\title{
Aprendizagem de estudantes do ensino técnico brasileiro: motivos, investimento e satisfação'
}

\author{
Marisa Aghetoni Fontes ${ }^{2}$ \\ ORCID: 0000-0003-4362-2566 \\ António Manuel Duarte ${ }^{3}$ \\ ORCID: 0000-0002-9497-7204
}

\section{Resumo}

0 estudo exploratório e qualitativo do qual este artigo faz um recorte teve como objetivo caracterizar a motivação para a aprendizagem de estudantes do ensino médio técnico brasileiro, no enquadramento da teoria Students' Approaches to Learning (SAL). Participaram 20 estudantes com idades entre 16 e 18 anos, que cursavam, em 2014, o primeiro ano do Ensino Médio Técnico do Instituto Federal de Educação, Ciência e Tecnologia do Rio de Janeiro, Campus Rio de Janeiro, os quais foram inquiridos sobre as suas orientações motivacionais para a aprendizagem. Para a recolha dos dados foram realizadas entrevistas semiestruturadas com base em um roteiro orientado para várias dimensões da orientação motivacional, nas quais este artigo incide: Intenção (motivos pessoais da aprendizagem), Investimento (quantidade de energia normalmente empregada na aprendizagem) e Satisfação (grau usual de satisfação com a aprendizagem). As respostas às entrevistas foram submetidas a uma análise de conteúdo temática. Os resultados são consistentes com a imagem sobre a variação da orientação motivacional dos estudantes para a aprendizagem em geral, fornecida pela teoria $S A L$, ou seja, motivação instrumental, motivação intrínseca e motivação de realização. Apresenta, porém, alguma especificidade e novidade, revelada pelo aparecimento de novas subvariantes dessas orientações, assim como de um padrão degradado de motivação. Os resultados são discutidos à luz das características dos estudantes inquiridos e das particularidades do ensino técnico brasileiro.

\section{Palavras-chave}

Motivação para a aprendizagem - Motivação do estudante - Ensino técnico.

\footnotetext{
1- Agradecimentos à Coordenação de Aperfeiçoamento de Pessoal de Nível Superior (CAPES) e ao Instituto Federal do Rio de Janeiro (IFRJ). 2- Instituto Federal de Educação, Ciência e Tecnologia do Rio de Janeiro IFRJ, Rio de Janeiro - RJ, Brasil. Contato: marisafontes@gmail.com. 3- Universidade de Lisboa, Lisboa - Portugal. Contato: amduarte@psicologia.ulisboa.pt.
} 


\section{Students' Learning in Brazilian Vocational Education: motives, investment, and satisfaction}

\section{Abstract}

The exploratory and qualitative study from which this article has been cut had the objective of characterizing the motivation for learning by Brazilian Vocational Education students, within the framework of the Students' Approaches to Learning (SAL) theory. A total of 20 junior students, aged 16 to 18 years, enrolled in Vocational Education at the Federal Institute of Education, Science, Technology of Rio de Janeiro, located in the same city in 2014 took part in the study and were asked about their motivational orientation for learning. In order to collect the data, semi-structured interviews were conducted based on a script looking at several dimensions of motivational orientation, including: Intention (personal reasons for learning), Investment (amount of energy usually used in learning), and Satisfaction (degree of satisfaction with learning). The answers to the interviews were submitted to a thematic content analysis. The results are consistent with the image about the variation of the students' motivational orientation towards learning in general, provided by the SAL theory, that is, instrumental motivation, intrinsic motivation and achievement motivation. However, they show some specificity and novelty, revealed by new sub-variants of those variants as well as a degraded pattern of motivation. The results are discussed in light of the characteristics of the students interviewed and the particularities of Brazilian Vocational Education.

\section{Keywords}

Motivation for learning - Student motivation - Vocational education.

\section{Introdução}

Este artigo é um recorte de uma investigação cujo tema é a motivação para a aprendizagem dos estudantes do ensino técnico brasileiro, tendo como referencial a teoria das Abordagens dos Estudantes à Aprendizagem - SAL (do inglês, Students' Approaches to Learning). Efetivamente, os estudos empíricos enquadrados nessa teoria (BEYAZATAS; SENEMOGLU, 2015; BIGGS, 1987; RICHARDSON, 2015) sugerem uma conceitualização do estudo acadêmico em termos da integração entre as suas orientações motivacionais e as suas respectivas estratégias utilizadas pelos estudantes - integração esta na base da variável composta designada de abordagem à aprendizagem. De acordo com esse referencial, considera-se como fundamental o estudo do fenômeno da aprendizagem acadêmica tendo como enfoque a experiência que os estudantes têm da mesma, ou seja, o modo como a vivenciam, ao invés de a considerar de um ponto de vista externo, ou seja, com base em observações do exterior. 
Especificamente no contexto brasileiro, as investigações acerca da aprendizagem são escassas dentro desse nível de ensino (SCACCHETTI; OLIVEIRA; RUFINI, 2014). Além disso, são também escassas nesse contexto quando consideramos o referencial teórico escolhido. São conhecidos dentro da teoria $S A L$ os estudos de Gomes (2010, 2011, 2013); Gomes; Golino (2012); Gomes et al. (2011); Galvão; Câmara; Jordão (2012); e Costa; Pfeuti; Casa Nova (2014). Não encontramos estudos na área dirigidos especificamente para os estudantes do ensino técnico brasileiro público e privado, que sofrem de altas taxas de abandono escolar (LINKE; NOGUEIRA, 2017; LÜSCHER; DORE, 2011; MEIRA, 2015).

Em termos gerais, a motivação consiste em um estado interno que ativa, orienta e mantém o comportamento do indivíduo em direção a um objetivo, sendo possível caracterizá-la em termos quer da causa, do conteúdo, da velocidade de iniciação e da intensidade desse comportamento, como da experiência cognitiva e emocional durante o envolvimento (DUARTE, 2012a; PEREIRA, 2013). No enquadramento da teoria SAL, a investigação visa a discriminar diferentes tipos de motivação (orientações motivacionais) dos estudantes para a aprendizagem: instrumental, intrínseca e de realização (BIGGS, 1987; BIGGS; TANG, 2011; FONTES, 2016; FONTES; DUARTE, 2017), a seguir caracterizadas pela relevância para os resultados, depois apresentados em termos de três das dimensões referidas: Intenção, Investimento e Satisfação.

A motivação instrumental para a aprendizagem refere-se a uma intenção de evitamento do fracasso, por meio de uma correspondência mínima às exigências, com reduzido investimento de energia e de tempo, visto que é dado pouco valor e significado ao conteúdo da aprendizagem, que é tido como desinteressante (DONNISON; PENNEDWARDS, 2012). 0 conteúdo a ser aprendido é tido como imposto externamente, sem relação com os interesses pessoais e proporcionando pouca satisfação (DUARTE, 2002). Esse tipo de motivação associa-se à intenção de acumular conhecimento, sem preocupação em compreendê-lo ou relacioná-lo com conhecimento prévio (LOURENÇO; PAIVA, 2015).

A motivação intrínseca para a aprendizagem caracteriza um envolvimento em função da satisfação com aquela aprendizagem, tida como forma de atualização de interesses e/ou competências e de desenvolvimento pessoal (DONNISON; PENN-EDWARDS, 2012; BIGGS; TANG, 2011). Com esse tipo de motivação, o estudante investe elevada energia para aprender, dedica mais tempo ao estudo e tende a confrontar ativamente as tarefas acadêmicas (DUARTE, 2012a; LOURENÇO; PAIVA, 2015; RICHARDSON, 2015). 0 foco de interesse recai assim sobre os significados subjacentes, as ideias principais, os temas e os princípios, utilizando-se evidências e aplicando-se o conhecimento em diferentes contextos, inclusive na vida quotidiana (BIGGS; TANG, 2011; DONNISON; PENNEDWARDS, 2012).

Como exemplo de uma motivação intrínseca, temos aqueles estudantes que se interessam em estudar pelo gosto em relação ao conhecimento, por gostarem de uma área específica ou por sentirem prazer em adquirir conhecimento sobre determinado conteúdo (FONTES, 2016). Esse tipo de motivação deriva, entre outras coisas, em uma compreensão mais complexa do tema a ser aprendido, bem como em um investimento na tarefa de aprendizagem e, potencialmente, no tema em si (PLATOW; MAVOR; GRACE, 2013). Por tudo isso, Biggs; Tang (2011) criaram uma metáfora para definir a motivação intrínseca: o valor na motivação intrínseca está na viagem e não no destino da mesma. 
Por seu lado, a motivação de realização para a aprendizagem caracteriza a procura de obtenção de classificações ou resultados elevados, no sentido de fortalecer o autoconceito acadêmico por meio da exibição da excelência pessoal (DUARTE, 2012a; LOURENÇO; PAIVA, 2015). Esse tipo de motivação envolve assim um comportamento competitivo e orientado para a autovalorização, independentemente dos conteúdos a aprender terem ou não significado pessoal (LOURENÇO; PAIVA, 2015). Nesse sentido, a satisfação está aqui relacionada à imagem pessoal e não à aprendizagem em si (LOURENÇO; PAIVA, 2015).

Pode-se inferir a respeito da frequência relativa dessas orientações motivacionais considerando os resultados de estudos que investigaram a representatividade das diferentes abordagens à aprendizagem que, como referido, envolvem as orientações motivacionais e as estratégias de aprendizagem. Na maior parte desses estudos, a abordagem de superfície à aprendizagem (que envolve uma motivação instrumental) surge como a mais comum entre os estudantes, em comparação com as restantes abordagens, de profundidade e de organização (que, correspondentemente, envolvem uma motivação intrínseca e de realização) (BOWDEN; ABHAYAWANSA; MANZIN, 2015; CHOY; DELAHAYE, 2012; MCDOWALL; JACKLING; NATOLI, 2015; VELO0; KRISHNASAMY; HARUN, 2015). A abordagem de superfície pode assim ser tida (GIBBS, 1992) como a abordagem mais normal e atipicamente esperada. Destaque-se que o mesmo resultado foi encontrado no estudo de Choy; Delahaye (2012) que, entre outras variáveis, investigou as abordagens da aprendizagem de estudantes do ensino técnico.

0 objetivo principal do presente estudo, de caráter exploratório e qualitativo, foi investigar as orientações motivacionais para a aprendizagem dos estudantes do ensino técnico brasileiro, na perspectiva da teoria $S A L$. Como objetivos específicos pretendeu-se, em primeiro lugar, caracterizar as variações de diferentes dimensões daquela orientação motivacional nestes estudantes, tal como identificadas por uma revisão de literatura sobre as abordagens à aprendizagem (DUARTE, 2002): Intenção (motivos pessoais da aprendizagem); Investimento (quantidade de energia normalmente empregada na aprendizagem); Percepção da tarefa (percepção usual das tarefas de aprendizagem); Avaliação do tempo (valorização habitual do tempo empregado na aprendizagem); Satisfação (grau usual de satisfação com a aprendizagem) e Contexto preferido (contexto preferido de aprendizagem). Em segundo lugar, pretendeu-se estudar a incidência das variações dessas dimensões das orientações motivacionais. Por limitações de espaço, este artigo incide apenas nas dimensões Intenção, Investimento e Satisfação.

\section{Método}

\section{Participantes}

0 estudo envolveu 20 estudantes, 10 do sexo feminino e outros 10 do sexo masculino, com idades entre 16 e 18 anos $(M=16,5 ; D P=0,77)$. Esses estudantes frequentavam o primeiro ano (segundo período) de um curso de Ensino Médio Técnico do Instituto Federal de Educação, Ciência e Tecnologia do Rio de Janeiro, Campus Rio de Janeiro, localizado na cidade do Rio de Janeiro, no ano de 2014. Eles frequentavam os cursos de Alimentos 
(20\%), Biotecnologia (20\%), Farmácia (20\%), Meio-Ambiente (20\%) e Química (20\%). No sentido de hipoteticamente aumentar a variabilidade das respostas dos participantes entrevistados, tentou-se diversificá-los no que diz respeito ao sucesso escolar, considerando que esse tende a variar com a motivação para a aprendizagem (DONNISON; EDWARDS, 2012; GIJBELS et al., 2005; VALADAS, 2014): 13 foram os alunos do curso com as médias escolares mais elevadas no período anterior $(M=8,2$ em uma escala de 0 a 10) e sete foram os alunos do curso com as médias escolares mais reduzidas no mesmo período $(M=4,5)$.

A amostra foi recolhida por conveniência e o critério de amostragem, para determinação da sua amplitude, foi o da saturação das categorias emergentes da análise de conteúdo temática realizada às respostas da entrevista.

\section{Contexto}

Com base em uma análise documental das matrizes curriculares e regimentos do Instituto, assim como de uma entrevista à sua diretora, procurou-se caracterizar o contexto de aprendizagem dos participantes. As competências por ele alvejadas são: habilidades exigidas no mercado de trabalho; postura crítica diante do trabalho a ser executado e da sociedade; conhecimento geral da sociedade; disciplina; responsabilidade e postura ética. Em âmbito cognitivo, as competências a desenvolver são: fundamentação teórica e prática do conhecimento; postura crítica e reflexiva diante do conhecimento e do trabalho a ser executado; conhecimento do funcionamento da sociedade; gestão e liderança. Na esfera comportamental, a competência a ser desenvolvida é a de conciliar a liberdade dada no contexto com a responsabilidade de organização pessoal para a aprendizagem e o aproveitamento.

Os objetivos educacionais são os de formar pessoas críticas, avaliadoras de possibilidades/alternativas em seus trabalhos, com conhecimento bem fundamentado, questionadoras/reflexivas, com responsabilidade e ética e capazes de aplicar o conhecimento ao seu trabalho, em paralelo com a compreensão daquilo que está sendo feito. No primeiro ano (o frequentado pelos participantes), o currículo é composto por disciplinas de formação geral, comuns ao ensino médio (Matemática, Química, Língua Portuguesa, Geografia, Biologia). A partir do primeiro ano, o currículo enfatiza a formação técnica, com disciplinas especializadas (Química Analítica, Físico-Química, Biossegurança, Estatística). 0 método de ensino envolve sobretudo aulas expositivas e aulas práticas de laboratório, mas o professor tem liberdade para decidir sobre o método a utilizar, de acordo com sua preferência ou com as necessidades da turma.

A mesma liberdade aplica-se ao método de avaliação, mas a maior parte dos professores optam pela utilização de provas. A avaliação é quantitativa (escala de 0 a 10), atendendo aos resultados na avaliação, mas também ao processo de aprendizagem. A concepção de aprendizagem vigente no contexto representa aquela como compreensão e memorização de informação através da aplicação refletida do conhecimento. A expectativa da escola sobre o processo de aprendizagem dos seus alunos envolve a aspiração de que aquele se opere pela experiência prática, no sentido da compreensão. 


\section{Recursos/Instrumentos}

Como instrumento de coleta de dados foi utilizado um roteiro adaptado (confira anexo) de um outro, pré-existente, a respeito das abordagens à aprendizagem, que tem uma parte com questões específicas sobre orientações motivacionais para a aprendizagem (DUARTE, 2012b) e que, entre outros, tem um enfoque nas dimensões da orientação motivacional aqui consideradas: Intenção (motivos pessoais da aprendizagem), Investimento (quantidade de energia normalmente empregada na aprendizagem) e Satisfação (grau usual de satisfação com a aprendizagem). A adaptação do roteiro pré-existente consistiu em especificar tanto os seus objetivos de avaliação (em cada uma das dimensões), como as suas questões para a aprendizagem no ensino técnico e em adequar a sua linguagem (português de Portugal) ao português do Brasil. 0 roteiro foi testado com dois estudantes do mesmo contexto educacional e modificado em função dos problemas detectados.

\section{Procedimentos}

Previamente à coleta dos dados, o estudo foi aprovado pela comissão de deontologia de uma faculdade de psicologia e foi autorizado pela direção geral da instituição estudada.

De modo a colher os dados, para uma caracterização das orientações motivacionais dos participantes para a aprendizagem no ensino técnico, foram feitas 20 entrevistas semiestruturadas na instituição frequentada pelos participantes, de acordo com o roteiro de entrevista com o consentimento informado desses, o dos seus pais ou responsáveis e o da direção geral da escola. As entrevistas foram realizadas individualmente, tendo sido gravadas em áudio e transcritas com a permissão dos participantes.

\section{Análise dos dados}

Após a transcrição, as respostas às entrevistas foram sujeitas a uma análise de conteúdo temática (MILES; HUBERMAN, 1994). Em uma primeira fase, o texto das respostas transcritas de cada entrevista foi segmentado dedutivamente em unidades temáticas (FLORES, 1994), de acordo com as dimensões consideradas no roteiro de entrevista, mas levando em conta o contexto global da entrevista. No sentido de validar essa segmentação, $20 \%$ das entrevistas foram segmentadas independentemente por outro analista. 0 grau de acordo interanalistas para a segmentação foi de 84,61\% para a dimensão Intenção, 87,5\% para a dimensão Investimento e 87,5\% para a dimensão Satisfação ${ }^{4}$.

Em uma segunda fase, cada uma das unidades temáticas segmentadas foi categorizada indutivamente, classificando-a em um tema específico de um sistema que foi evoluindo ao longo da análise. No final, com o sistema de categorias emergentes construído, essas foram organizadas em metacategorias e novamente categorizadas de acordo com esse sistema. Para validar a categorização, todas as entrevistas foram categorizadas independentemente por um analista independente, treinado para esse fim. 0 grau de acordo interanalistas

4- Para todos os cálculos, foi utilizada a fórmula sugerida por Bakeman; Gottman (1986): $\mathrm{PA}=\mathrm{Na} /(\mathrm{Na}+\mathrm{Nd})$ * 100; onde "PA" é a percentagem de acordo; "Na" é a frequência dos acordos; "Nd" é a frequência dos desacordos. 
para a categorização foi de 93,55\% em geral, de 85,71\% para a dimensão Intenção e 100\% tanto para a dimensão Investimento como para a dimensão Satisfação.

Para a exploração das categorias e metacategorias, foi analisada a frequência relativa de cada uma delas na amostra de participantes, depois de resolvidos os desacordos registrados entre os dois analistas. Essa representatividade foi calculada contabilizando a presença de cada categoria e meta-categoria no discurso de cada participante, considerando apenas uma incidência da respectiva categoria e meta-categoria, independentemente do número de vezes em que ela esteve presente naquele discurso. Foi utilizado o programa de informática NVivo - versão 10 para apoio a esta análise.

Foi analisada também a relação das categorias e das metacategorias entre si, pelo estudo da sua co-ocorrência no discurso dos participantes (contabilizou-se a presença de cada categoria e metacategoria no discurso de cada participante considerando apenas uma incidência da respectiva categoria e meta-categoria, independentemente do número de vezes em que ela esteve presente naquele discurso). Essa análise, apoiada pelo programa informático SPSS - versão 24, foi efetuada por meio de tabelas de contingência e teste de Qui-quadrado de independência ou teste de Fisher (para os casos em que em alguma das células se esperava uma contagem menor que 5).

\section{Resultados}

A análise das respostas às entrevistas permitiu chegar a um sistema de categorias (e, nalgumas dimensões da motivação, de meta-categorias), descritivas de diferentes tipos de motivo, investimento e satisfação na aprendizagem nos estudantes de Ensino Técnico inquiridos. Os resultados relativos à dimensão motivacional Intenção, revelaram a existência de quatro tipos de motivação (meta-categorias): motivação intrínseca, motivação de realização, motivação instrumental e padrão degradado de motivação. A primeira meta-categoria, motivação intrínseca ( $n=13 ; 65 \%)$, implica o envolvimento na aprendizagem em função da satisfação em relação a ela, tida como forma de atualização de interesses de desenvolvimento pessoal. Abrange duas categorias: aplicar e retirar prazer da aprendizagem. A categoria aplicar $(n=5 ; 25 \%)$, diz respeito a uma motivação para aplicar, na prática, as aprendizagens realizadas (“(...) [aprendo para] botar em prática logo, trabalhar logo, atuar logo.”). A categoria retirar prazer da aprendizagem ( $n=12 ; 60 \%)$, diz respeito a uma motivação de retirar prazer do conteúdo aprendido ("Digamos que [aprendo porque] eu gosto da área que eu estou estudando."). A segunda meta-categoria, motivação de realização $(n=15 ; 75 \%)$, caracteriza-se pela procura de obtenção de classificações ou resultados elevados, no sentido de fortalecer o autoconceito acadêmico através da exibição da excelência pessoal. Abrange cinco categorias: superior, trabalho, realização, classificações e certificação. A categoria superior ( $n=5 ; 25 \%$ ), refere-se a uma motivação de poder prosseguir para o Ensino Superior ("[a minha intenção para a aprendizagem é] dar uma base muito grande pra minha faculdade e para eu passar também.”). A categoria trabalho $(n=10 ; 50 \%)$, diz respeito a uma motivação de poder ingressar no mercado de trabalho (“(...) [aprender] dá a oportunidade de arrumar um emprego mais rápido mais tarde.”). A categoria realização $(n=3 ; 15 \%)$, diz respeito a uma 
motivação de vencer as dificuldades de aprender uma área de estudo considerada como difícil e desafiante ("eu acho difícil e é por causa disso que eu gosto de Química porque quando você tem alguma coisa que te desafia, eu me sinto mais motivada para fazer aquilo.”). A categoria classificações ( $n=2 ; 10 \%)$, diz respeito a uma motivação de obter classificações elevadas (“[a minha intenção para a aprendizagem é] Ter boas notas(...).”). A categoria certificação $(n=4 ; 20 \%)$, diz respeito a uma motivação de obter uma certificação acadêmica (“ (...) [aprendo para] conseguir realizar o curso bem (...) ter um diploma.”). A terceira meta-categoria, motivação instrumental $(n=3 ; 15 \%)$, está relacionada à procura de evitamento do fracasso, através de uma correspondência mínima às exigências, visto que o conteúdo da aprendizagem é tido como desinteressante, sem relação com os interesses pessoais, e como imposto exteriormente. Abrange três categorias: indicação, exemplo e evitar insucesso. A categoria indicação $(n=3 ; 15 \%)$, diz respeito a aquiescer à indicação de alguma pessoa para estudar no Ensino Técnico ("entrei aqui [no Ensino Técnico] primeiro por questão de aconselhamento dos meus pais que o Ensino Técnico e Federal seria muito bom para mim."). A categoria exemplo $(n=1 ; 5 \%)$, diz respeito a seguir o exemplo de alguém na família que estuda ou estudou no Ensino Técnico ("meu irmão fez ensino [técnico e] federal, então eu tive aquele espelho nele, então eu queria fazer o mesmo.”). A categoria evitar insucesso $(n=1 ; 5 \%)$, diz respeito à motivação de obter classificações que permitam evitar o insucesso (“(...) [aprendo para] passar, não reprovar (...).”). A quarta e última meta-categoria, padrão degradado de motivação ( $n=2 ; 10 \%)$, refere-se a uma motivação para o convívio social na escola ou para o seu abandono. Abrange duas categorias: convívio e abandono. A categoria convívio $(n=2 ; 10 \%)$, diz respeito a uma motivação de convívio social com outras pessoas na escola (“(...) [aprendo] porque eu gosto do ambiente social proporcionado pelo colégio.”). A categoria abandono ( $n=1 ; 5 \%)$, diz respeito a uma motivação de cessar a aprendizagem abandonando a escola ("ao longo dos períodos eu fui me desgastando e perdeu essa motivação e eu fui vendo que eu não tenho muito a ver com o colégio.").

Por seu lado, os resultados referentes à dimensão motivacional Investimento, concernente à quantidade de energia normalmente despendida na aprendizagem no Ensino Técnico, revelaram a existência de três categorias: elevado, mediano e reduzido. A categoria elevado $(n=12 ; 60 \%)$, diz respeito a uma elevada quantidade de energia investida na aprendizagem ("[invisto] Muita energia [na aprendizagem].”). A categoria mediano $(n=7 ; 35 \%)$, refere-se a uma mediana quantidade de energia investida na aprendizagem ("eu acho que [invisto uma energia] média [na aprendizagem]."). A categoria reduzido $(n=5 ; 25 \%)$, identifica uma reduzida quantidade de energia investida na aprendizagem ("eu acho que eu gasto muito pouca energia [na aprendizagem].”.

Os resultados relativos à dimensão motivacional Satisfação, que diz respeito ao nível de satisfação em relação à aprendizagem no Ensino Técnico, revelaram a existência de quatro categorias: alta, média-alta, média e baixa. A categoria alta ( $n=15 ; 75 \%)$, diz respeito a um nível alto de satisfação com a aprendizagem ("estou bem satisfeita com o que eu estou aprendendo.”). A categoria média-alta ( $n=6 ; 30 \%)$, identifica um nível médioalto de satisfação com a aprendizagem ("[a minha satisfação com a aprendizagem] é uma satisfação de média para alta.”). A categoria média $(n=1 ; 5 \%)$, refere-se a um nível médio 
de satisfação com a aprendizagem ("eu diria [que a minha satisfação com a aprendizagem é] média.”). A categoria baixa ( $n=1 ; 5 \%)$, diz respeito a um nível baixo de satisfação com a aprendizagem (“[a minha satisfação com a aprendizagem é] baixa.”).

Finalmente, o estudo da coocorrência das meta-categorias e categorias detectadas permitiu constatar a existência de uma relação positiva e significativa entre a categoria retirar prazer da aprendizagem (motivação para retirada de prazer do conteúdo aprendido) e a meta-categoria motivação intrínseca (envolvimento na aprendizagem em função da satisfação em relação a ela, tida como forma de atualização de interesses de desenvolvimento pessoal) ( $p=0,00$ ), que coocorrem em 60\% dos casos; e entre a categoria trabalho (motivação para ingresso no mercado de trabalho) e a meta-categoria motivação de realização (obtenção de classificações ou resultados elevados, no sentido de fortalecer o autoconceito acadêmico através da exibição da excelência pessoal) $(p=0,03)$, que coocorrem em 50\% dos casos.

\section{Discussão}

Os resultados deste estudo são consistentes com a concepção sobre a motivação para a aprendizagem em geral fornecida pela teoria $S A L$, apresentando, porém, certa especificidade e novidade, de resto esperada considerando o contexto particular investigado. Os três primeiros tipos de motivação para a aprendizagem (dimensão Intenção) identificados nos estudantes de ensino técnico inquiridos (motivação intrínseca, motivação de realização e motivação instrumental) replicam os tipos de motivação para a aprendizagem identificados pela investigação realizada no enquadramento da teoria SAL (BEYAZTAS; SENEMOGLU, 2015; BIGGS, 1987; BIGGS; TANG, 2011; BOWDEN; ABHAYAWANSA; MANZIN, 2015; CHOY; DELAHAYE, 2012; DONNISON; PENN-EDWARDS, 2012; MCDOWALL; JACKLING; NATOLI, 2015).

A ocorrência desses três tipos de motivação pode explicar-se quer pela variabilidade das características pessoais dos estudantes inquiridos (ao nível das suas concepções e estratégias de aprendizagem), quer pela natureza do seu contexto institucional de aprendizagem. Considerando esta última, a motivação intrínseca, em particular, pode estar relacionada à ênfase aparentemente colocada por aquele contexto nos processos de compreensão, reflexão e crítica, com os quais aquele tipo de motivação tende a associarse. Por outro lado, hipotetiza-se que a presença da motivação de realização é coerente com o objetivo do contexto institucional em formar para posições de trabalho qualificado, cuja oportunidade, em um mercado de trabalho competitivo, dependerá do aproveitamento escolar. Por seu lado, a existência paralela da motivação instrumental pode estar relacionada à elevada carga de trabalho e exigência do contexto de aprendizagem, que assim poderá conduzir estudantes menos habituados a essas condições a uma abordagem de superfície à aprendizagem (em que está envolvida aquela motivação).

0 quarto tipo de motivação para a aprendizagem aqui identificado (o padrão degradado de motivação, que se refere a uma motivação para o convívio social na escola ou para o seu abandono) parece divergir e apresentar-se como novidade em relação ao mapeamento da motivação realizado no quadro da teoria $S A L$. Se uma motivação para o 
convívio não é incompativel com outros tipos de motivação (motivação de realização), já uma motivação de abandonar a escola pode, hipoteticamente, dever-se uma ausência de identificação com a falta de interesse para aprender os conteúdos curriculares abordados no curso que se frequenta.

Note-se que o ingresso nos Institutos Federais brasileiros de ensino técnico depende de um processo seletivo muito concorrido e que os estudantes que conseguem ingressar nos cursos mais procurados do ensino médio Técnico são aqueles com as melhores notas e, provavelmente, provenientes de escolas particulares (SANTOS; SANTOS, 2016). Sendo assim, os estudantes com piores classificações, mesmo que tenham optado por estudar em um desses cursos, acabam por ter de estudar nos demais. Esse fato pode desestimular os alunos, como relatado em algumas entrevistas. No entanto, nem todos os alunos revelam uma vontade efetiva de abandonar o ensino técnico, mas sim, o curso que frequentam, apresentando como solução tentar uma transferência de curso nos próximos períodos letivos. Por outro lado, essa orientação motivacional pode estar relacionada com as particularidades do contexto de aprendizagem dos estudantes inquiridos, que pela exigência de um ritmo de estudo intenso, rigoroso e organizado, conforme revelado na entrevista da diretora da instituição, pode desmotivar estudantes com mais dificuldades em um envolvimento desse tipo.

Ainda no que diz respeito aos resultados da dimensão Intenção, considerando a representatividade dos tipos de motivação encontrados, a primazia da motivação de realização (em 75\% dos participantes), seguida de perto da motivação intrínseca (65\%) e, de longe, tanto da motivação instrumental (15\%) como do padrão degradado de motivação (10\%), não vai ao encontro à primazia de uma abordagem de superfície à aprendizagem (onde está implicada uma motivação instrumental), encontrada na maior parte dos estudos SAL no ensino técnico (BIGGS, 1982; 1987; CHOY; DELAHAYE, 2012; GIBBS, 1992), e no ensino médio e Superior (MCDOWALL; JACKLING; NATOLI, 2015). No entanto, os resultados alinham com resultados de estudos minoritários, como o de Beyaztas; Senemoglu (2015), que encontraram uma maior incidência da abordagem de organização (em que está implicada uma motivação de realização), seguida de uma abordagem de profundidade (em que está implicada uma motivação intrínseca) e de uma menor incidência da abordagem de superfície (em que está implicada uma motivação instrumental). A maior incidência registrada da motivação de realização pode relacionarse à ansiedade sobre o futuro, no sentido de alcançar uma boa posição no mercado de trabalho, a que muitos dos entrevistados com aquela motivação aludiram. No mesmo sentido concorrerá o fato do contexto de aprendizagem dos participantes parecer estar orientado para o seu sucesso acadêmico e profissional, no sentido de lhes proporcionar o ingresso em universidades ou posições de emprego de maior prestígio. Isso alinha-se com as conclusões do estudo de Beyaztas; Senemoglu (2015), no sentido de que quando o contexto educacional é baseado na competição e na expectativa de notas elevadas, a preferência dos estudantes vai para uma abordagem de organização (que envolve uma motivação de realização). Outra hipótese para o alto índice de motivação de realização pode estar relacionada com a eventualidade da maior parte dos estudantes de ensino técnico inquiridos trabalharem melhor sob pressão. 
Por seu lado, o fato de a motivação intrínseca ser a segunda mais presente entre os estudantes de ensino técnico pesquisados (65\%) alinha-se com os resultados do estudo de Ramsden (1983), igualmente com estudantes do ensino técnico, que constatou que esses estavam tipicamente interessados em perceber os conteúdos dos seus respectivos cursos encontrando-se bastante motivados para esse fim. 0 elevado índice de motivação intrínseca observado (assim como o reduzido índice de motivação instrumental) poderá ser igualmente dependente dos métodos de ensino aparentemente prevalentes no contexto do ensino técnico estudado.

Efetivamente, sabemos que um ensino centrado nos estudantes (BEYAZTAS; SENEMOGLU, 2015), que apresenta os conteúdos de forma clara e em relação com outros conteúdos (AZER; GUERRER0; WALSH, 2013), que fornece problemas e coloca questões (BIGGS; TANG, 2011) e que fornece alguma liberdade de escolha contribui para a utilização de uma abordagem de profundidade à aprendizagem (na qual está envolvida uma motivação intrínseca).

0 fato de a motivação instrumental estar sub-representada na amostra inquirida (15\%) articula-se com a expectativa, por parte do contexto de aprendizagem, de que poucos alunos apresentem aquele tipo de motivação, uma vez que ela é incompatível com o elevado nível de envolvimento e competitividade esperado nos alunos. Outra possível explicação é a de que a maior parte dos alunos que procuram esse tipo de contexto de aprendizagem, que conseguem superar o processo seletivo de ingresso e que conseguem permanecer nele, não possam apresentar uma motivação instrumental, pela incompatibilidade desta com aquele tipo de contexto. Apesar de envolver uma amostra muito reduzida, o presente estudo poderá assim indiciar que em contextos de formação profissionalizante, os estudantes não desenvolvem necessária e maioritariamente uma motivação instrumental, na linha de estudos anteriores (IYER; ROBERTS, 2014; MCDOWALL; JACKLING; NATOLI, 2015), que constataram o mesmo para a abordagem de superfície (que envolve aquele tipo de motivação).

A representatividade muito reduzida do padrão degradado de motivação (10\%) não coincide com as altas percentagens de abandono dos estudantes de ensino técnico no Brasil, apontadas como em grande parte resultantes da desmotivação dos alunos (BASTOS; GOMES, 2014; LÜSCHER; DORE, 2011). 0 padrão degradado de motivação pode ser explicado pela referência, nos discursos de certos estudantes que apresentam as categorias convívio e abandono, à elevada exigência do contexto de aprendizagem, com elevada carga de conteúdos e horas de permanência em aulas e atividades, acrescido da obrigatoriedade de dedicação praticamente exclusiva à escola.

Passemos agora à discussão dos resultados relativos à dimensão Investimento, que diz respeito à quantidade de energia normalmente despendida na aprendizagem no ensino técnico. 0 investimento elevado foi igualmente encontrado em estudantes de música (ANASTÁCIO, 2013), teatro (TABORDA, 2015) e do $1^{\circ}$ Ciclo de escola básica (FIGUEIRA, 2017) e pode ser comparado ao investimento elevado implicado na motivação intrínseca para a aprendizagem em geral (BIGGS, 1987). 0 investimento mediano pode ser analisado à luz da explicação da maior parte dos estudantes que o apresentam, que entendem que face ao investimento que o ensino técnico demanda, eles dedicam-se bastante, mas não o suficiente. 
Por seu lado, o investimento reduzido provavelmente ocorre pela reduzida motivação para o estudo ou por comparação com o grau de investimento exigido pelo contexto.

A predominância do investimento elevado (60\%), seguida de longe pela do investimento mediano (35\%) e do reduzido (25\%), é convergente com o aparentemente elevado padrão de exigência por parte do contexto de aprendizagem dos participantes e com a elevada carga de trabalho que impõe.

Em relação aos resultados da dimensão Satisfação, que diz respeito à quantidade de satisfação que os estudantes apresentam na aprendizagem no ensino técnico, a variabilidade detectada (satisfação alta, média-alta, média e baixa) foi igualmente encontrada em outros estudos do referencial SAL (ANASTÁCIO, 2013; TABORDA, 2015). A satisfação em relação à aprendizagem está provavelmente associada às características de cada estudante (ENTWISTLE, 2005) e da percepção que esse faz do contexto pessoal (BIGGS, 1987), tendo alguns participantes relacionado aquela satisfação com o tipo de ensino e a carga de trabalho a que estão sujeitos.

Consideremos, por fım, os resultados referentes à relação entre os tipos de motivação para a aprendizagem detectados. A relação positiva e significativa entre o motivo de retirar prazer da aprendizagem (isto é, motivação de retirar prazer do conteúdo aprendido) e a motivação intrínseca é condizente com a teoria $S A L$, uma vez que, de acordo com ela, aquele tipo de motivação intrínseca para a aprendizagem é, em parte, caracterizada por um envolvimento na aprendizagem em função do prazer intrínseco que dela se retira (BIGGS; TANG, 2011; DONNISON; PENN-EDWARDS, 2012; DUARTE, 2002). No mesmo sentido, a relação positiva e significativa entre o motivo trabalho (isto é, motivação para poder ingressar no mercado de trabalho) e a motivação de realização está igualmente em consonância com a teoria $S A L$, uma vez que, de acordo com ela, a motivação de realização para a aprendizagem caracteriza um envolvimento em função de possiveis ganhos que lhe são extrínsecos (DUARTE, 2002; 2012a; LOURENÇO; PAIVA, 2015).

\section{Conclusão}

Consideramos que este estudo acerca da motivação para a aprendizagem dos estudantes do ensino técnico, na perspectiva da teoria $S A L$, constitui uma contribuição para o conhecimento desta população. Lembremos que as altas taxas de abandono e evasão no ensino técnico brasileiro têm como um dos principais fatores problemas na motivação para a aprendizagem (LINKE; NOGUEIRA, 2017). Na linha do referencial $S A L$, o estudo permitiu ainda considerar o fenômeno da motivação para a aprendizagem no ensino técnico tendo como enfoque de investigação a experiência dos estudantes, ao invés de considerar a aprendizagem do ponto de vista de um observador externo, como na maior parte das investigações anteriores sobre o tema.

Este estudo sugere que tal como o investimento e a satisfação na aprendizagem, os motivos dos estudantes inquiridos do ensino técnico brasileiro podem, em parte, variar do mesmo modo que variam os motivos para a aprendizagem em geral, tal como apurado pela teoria $S A L$, ou seja, em termos de uma motivação instrumental, intrínseca e, principalmente, de realização. No entanto, esta replicação não é totalmente isomórfica, 
considerando a observação, neste estudo, quer de novas subvariantes de cada um desses tipos de motivação, quer de um padrão degradado de motivação.

No entanto, os resultados devem ser considerados com cautela, considerando as limitações do estudo realizado, sobretudo relacionadas com a reduzida amostra de participantes nele envolvidos, de um único ano de escolaridade, da mesma instituição e avaliados através das suas auto-observações. São assim necessários estudos futuros sobre a motivação dos estudantes de ensino técnico com amostras mais amplas e diversificadas e que utilizem uma variedade de métodos de avaliação.

Para além disso, são necessários estudos longitudinais, que investiguem a variação da motivação ao longo do ensino técnico; estudos comparativos, que diferenciem a motivação de estudantes desse contexto com diferentes níveis de aproveitamento; e estudos que testem o efeito de intervenções dirigidas à modificação da motivação para a aprendizagem no ensino técnico.

Finalmente, do ponto de vista das implicações práticas, este estudo sugere a necessidade de diagnosticar e inverter, nos estudantes de ensino técnico, eventuais níveis reduzidos de investimento e satisfação na aprendizagem, uma eventual motivação instrumental e um eventual padrão degradado de motivação, tendo em conta os seus efeitos negativos no sucesso escolar. Tal ação poderá ser feita por meio de intervenções centradas tanto nos estudantes de ensino técnico como no seu contexto de aprendizagem que, igualmente, proporcionem aumento e integração da eventual motivação intrínseca com a eventual motivação de realização. Além disso, que considerem os seus efeitos positivos tanto no sucesso e na qualidade da aprendizagem, como na integração no mercado de trabalho.

\section{Referências}

ANASTÁCI0, Sara. Orientações motivacionais para a aprendizagem em estudantes de música. 2013. Dissertação (Mestrado em Psicologia) - Faculdade de Psicologia, Universidade de Lisboa, Lisboa, 2013.

AZER, Sammy; GUERRER0, Anthony; WALSH, Allyn. Enhancing learning approaches: practical tips for students and teachers. Medical Teacher, Dundee, v. 35, n. 6, p. 433-443, 2013.

BAKEMAN, Roger; GOTTMAN, John. Observing interaction: an introduction to sequential analysis. New York: Cambridge University Press, 1986.

BASTOS, Oliver Guimarães; GOMES, Carlos Francisco Simões. A evasão escolar no ensino técnico: entendendo e enfrentando as dificuldades - Um estudo de caso do CEFET-RJ. In: CONGRESSO NACIONAL DE EXCELÊNCIA EM GESTÃO, 10., 2014, Rio de Janeiro. Anais... Rio de Janeiro: Firjan, 2014. p. 1-13.

BEYAZTAS, Dilek; SENEMOGLU, Nuray. Learning approaches of successful students and factors affecting their learning approaches. Education \& Science / Egitim Ve Bilim, Ancara, v. 40, n. 179, p. 193-216, 2015.

BIGGS, John. Student motivation and study strategies in university and college of advanced education populations. Higher Education Research \& Development, v. 1, n. 1, p. 33-55, 1982. 
BIGGS, John. Student approaches to learning and studying. Hawthorn: Australian Council for Educational Research, 1987.

BIGGS, John; TANG, Catheryne. Teaching for quality learning at university. 4. ed. New York: McGraw-Hill; Maidenhead: Open University Press, 2011.

BOWDEN, Mark; ABHAYAWANSA, Subhash; MANZIN, Gregoria. A multiple cross-cultural comparison of approaches to learning. Compare, London, v. 45, n. 2, p. 272-294, 2015.

CHOY, Sarojni; DELAHAYE, Brian. Learning approaches, study orientation and readiness for self-directed learning of youth in TAFE. In: VOCATIONAL EDUCATION AND TRAINING RESEARCH CONFERENCE, 2012, Harbour. Anais... Harbour: Coffs Harbour, 2012. p. 1-14.

COSTA, Simone Alves da; PFEUTI, Maria de la Mercedes; CASA Nova, Silvia Pereira de Castro. As estratégias de ensino-aprendizagem utilizadas pelos docentes e sua relação com o envolvimento dos alunos. Revista Evidenciação Contábil \& Finanças, João Pessoa, v. 2, n. 1, p. 59-74, 2014.

DONNISON, Sharn; PENN-EDWARDS, Sorrel. Focusing on first year assessment: surface or deep approaches to learning? The International Journal of the First Year in Higher Education, Brisbane, v. 3, n. 2, p. 9-20, 2012.

DUARTE, António. Aprender melhor: aumentar o sucesso e a qualidade da aprendizagem. Lisboa: Escolar, 2012a.

DUARTE, António. Aprendizagem, ensino e aconselhamento educacional. Porto: Porto Editora, 2002.

DUARTE, António. Guião de entrevista da abordagem à aprendizagem. Lisboa: Universidade de Lisboa, 2012b. Documento interno da Faculdade de Psicologia da Universidade de Lisboa.

ENTWISTLE, Noel. Learning outcomes and ways of thinking across contrasting disciplines and settings in higher education. The Curriculum Journal, London, v. 16, n. 1, p. 67-82, 2005.

FIGUEIRA, Ana Isa. Intenções, maneiras e ideias sobre 0 aprender na escola básica: abordagens à e conceções de aprendizagem em alunos do $1 .{ }^{\circ}$ ciclo de escolaridade: relações com o contexto. 2017. Tese (Doutorado em Psicologia) - Faculdade de Psicologia, Universidade de Lisboa, Lisboa, 2017.

FLORES, Javier Gil. Análisis de datos cualitativos: aplicaciones a la investigación educativa. Barcelona: PPU, 1994.

FONTES, Marisa Aguetoni. Memorização e leitura dinâmica. 3. ed. Rio de Janeiro: Ferreira, 2016.

FONTES, Marisa Aghetoni; DUARTE, António. Motivos para a aprendizagem em estudantes do ensino técnico brasileiro. In: FONTES, Marisa Aghetoni (Org.). Pesquisar psicologia. Lisboa: Chiado, 2017. p. 203-231.

GALVÃO, Afonso; CÂMARA, Jacira; JORDÃO, Michelle. Estratégias de aprendizagem: reflexões sobre universitários, Revista Brasileira de Estudos Pedagógicos, Brasília, DF, v. 93, n. 235, p. 627-644, 2012.

GIBBS, Graham. Improving the quality of student learning: theory and practice. Bristol: TES, 1992. 
GIJBELS, David et al. Effects of problem based learning: a meta-analysis from the angle of assessment. Review of Educational Research, Pensilvania, v. 75, n. 1, p. 27-61, 2005.

GOMES, Cristiano Assis. A construção de uma medida em abordagens de aprendizagem. Psico, Porto Alegre, v. 44, p. 193-203, 2013.

GOMES, Cristiano Assis. Perfis de estudantes e a relação entre abordagens de aprendizagem e rendimento escolar. Psico, Porto Alegre, v. 41, n. 4, p. 503-509, 2010.

GOMES, Cristiano Assis. Validação da escala de abordagens de aprendizagem (EABAP) em uma amostra brasileira. Psicologia, Porto Alegre, v. 24, p. 19-27, 2011.

GOMES, Cristiano Assis; GOLINO, Hudson. Validade incremental da escala de abordagens de aprendizagem (EABAP). Psicologia Reflexão e Crítica, Porto Alegre, v. 25, n. 4, p. 623-633, 2012.

GOMES, Cristiano Assis et al. Validação da escala de abordagens de aprendizagem (EABAP) em uma amostra brasileira. Psicologia, Porto Alegre, v. 24, n. 1, p. 19-27, 2011.

IYER, Ashok; ROBERTS, Andrews. A phenomenographic study in understanding architecture students' approaches to learning the coursework of architectural design. The Higher Education Academy, New York, v. 9, n. 1, p. 89-109, 2014

LINKE, Elizandra Campos; NOGUEIRA, Bárbara Campos. A evasão escolar no ensino técnico profissionalizantes. In: SEMINÁRIO INTERINSTITUCIONAL DE ENSINO, PESQUISA E EXTENSÃO, 22., 2017. Cruz Alta. Anais... Cruz Alta: Unicruz, 2017. p. 1-14.

LOURENÇO, Abílio Afonso; PAIVA, Maria Olímpia. Abordagens à aprendizagem: a dinâmica para o sucesso académico. CES Psicologia, Julz de Fora, v. 8, n.2, p. 47-75, 2015.

LÜSCHER, Ana Zuleima; DORE, Rosemary. Política educacional no Brasil: educação técnica e abandono escolar. Revista Brasileira de Pós Graduação, Brasília, DF, v. 8, n. 1, p. 147-176, 2011.

MCDOWALL, Tracey; JACKLING, Beverley; NATOLI, Ricardo. Relationships between vocational interests and learning approaches to advance the quality of student learning in accounting. Accounting Education, DeKalb, v. 24, n. 6, p. 498-513, 2015.

MEIRA. Cristiane Araújo. A evasão escolar no ensino técnico profissionalizante: um estudo de caso no campus Cariacica do Instituto Federal do Espírito Santo. 2015. 118 f. Dissertação (Mestrado em Gestão Pública) Centro de Ciências Jurídicas e Econômicas da Universidade Federal do Espírito Santo, Vitória, 2015.

MILES, Matthew; HUBERMAN, Michael. Qualitative data analysis: an expanded sourcebook. 2. ed. California: Sage, 1994.

PEREIRA, Anabela. Motivação na aprendizagem e no ensino. In: VEIGA, Feliciano H. (Coord.). Psicologia da educação: teoria, investigação e aplicação, envolvimento dos alunos na escola. Lisboa: Climepsi, 2013. p. 445-493. 
PLATOW, Michael; MAVOR, Keneth; GRACE, Diana. On the role of discipline-related self-concept in deep and surface approaches to learning among university students. Instructional Science, Basileia, v. 41, n. 2 , p. 271-285, 2013.

RAMSDEN, Paul. Institutional variations in British students' approaches to learning and experiences of teaching. Higher Education, London, v. 12, n. 6, p. 691-705, 1983.

RICHARDSON, John. Approaches to learning or levels of processing: what did Marton and Säljö (1976a) really say? The legacy of the work of the Göteborg Group in the 1970s. Interchange, Heidelberg, v. 46, n.3, p. 239-269, 2015.

SANTOS, Danielle Sousa; SANTOS, Marcela Kleiciane Nascimento. Instituto Federal de Educação, Ciência e Tecnologia de São Paulo e o direito a uma escola pública de qualidade: um estudo sobre o perfil dos jovens estudantes ingressantes no ensino médio técnico integrado. Revista Brasileira da Educação Profissional e Tecnológica, Natal, v. 1, n. 7, p. 115-124, mar. 2016.

SCACCHETTI, Fabio Alexandre Pereira; OLIVEIRA, Katya Luciane de; RUFINI, Sueli Édi. Medida de motivação para aprendizagem no ensino técnico profissional. Avaliação Psicológica, Campinas, v. 13, n. 2, p. 297-305, 2014.

TABORDA, Pedro. Orientações motivacionais em estudantes de teatro: um estudo exploratório. 2015. Dissertação (Mestrado em Psicologia) - Faculdade de Psicologia, Universidade de Lisboa, Lisboa, 2015.

VALADAS, Sandra Santos. Abordagens ao estudo e sucesso académico no ensino superior. Revista Eletrónica de Psicologia, Educação e Saúde, Coimbra, v. 4, n. 1, p. 47-67, 2014.

VELO0, Arsaythamby; Krishnasamy, Hariharan; Harun, Hana Mulyani. What are the learning approaches applied by undergraduate students in English process writing based on gender? International Education Studies, Toronto, v. 8, n. 6, p. 46-55, 2015.

Recebido em: 08.03.2018 Revisões em: 16.05.2018 Aprovado em: 07.08.2018

Marisa Aghetoni Fontes é doutora em psicologia da educação pela Faculdade de Psicologia da Universidade de Lisboa, bolsista de doutorado pleno Coordenação de Aperfeiçoamento de Pessoal de Nível (CAPES). É mestre em psicologia escolar pelo Instituto de Psicologia da Universidade de São Paulo (IP/USP). É servidora pública no Instituto Federal de Educação, Ciência e Tecnologia do Rio de Janeiro.

António Manuel Duarte desempenha atividades de docência e investigação na área da psicologia educacional, paralelamente à da psicologia da arte na Faculdade de Psicologia da Universidade de Lisboa. Coordena o Programa de Investigação em Concepções de \& Abordagens à Aprendizagem. 